\title{
Selective degradation of styrene-contained plastics catalyzed by iron under visible light
}

\author{
Miao Wang, Jinglan Wen, Yahao Huang, Peng $\mathrm{Hu}^{*}$ \\ Lehn Institute of Functional Materials, School of Chemistry, Sun Yat-Sen University, \\ Guangzhou 510275, China \\ Email: hupeng8@mail.sysu.edu.cn
}

Efficient degradation of plastics, the vital challenge for a sustainable future, stands in need of better chemical recycling procedures that help produce commercially valuable small molecules and redefine plastic waste as a rich source of chemical feedstock. However, the corresponding chemical recycling methods, while being generally restricted to polar polymers, need improvement. Particularly, degradation of chemical inert nonpolar polymers, the major constitutes of plastics, are reported to have suffered from low selectivity and very harsh transformation conditions. Herein, we report an efficient method for the selective degradation of styrene-contained plastics under gentle conditions through oxidative multiple sp ${ }^{3}$ $\mathrm{C}-\mathrm{C}$ bond cleavage. The unpresented procedure is catalyzed with inexpensive iron salts under visible light, using oxygen as the green oxidant. Furthermore, simple iron salts can be used to degrade plastics in the absence of solvent under natural conditions, highlighting the potential application of iron salts as additives for degradable plastics.

Plastics, being indispensable for everyday life, have greatly benefited the modern world with their safety, convenience, and wide applicability. While being essential for advanced technology development, plastics, because of their economy in pricing, are facing the challenges brought by casual disposal and the consequent plastic pollution to the environment ${ }^{1-5}$. By 2015, around 6,300 million tons of plastic waste has been generated and expanded around the world, and the amount is quickly increasing every 
year ${ }^{5}$. In contrast to this, the current procedure of plastic production is still largely unsustainable, due to its reciprocal relation with the oil industry which is also consumptive and contaminative $\mathrm{e}^{2,6}$. Moreover, the inert nature of polymers, resulting in a long degradation period of several hundred years under natural conditions, is the fundamental reason for plastic pollution. Based on that, despite the developments of new sustainable plastics ${ }^{1,7-9}$, the relevant pollution remains a big challenge, so long as the existing plastic system stays regular in the foreseeable future ${ }^{2}$. To solve the problem, many methods have been tried on plastic recycling and degradation, for example, reuse of plastic that suffers from deterioration of material properties ${ }^{10}$; chemical recycling which transforms polymers into valuable small molecules ${ }^{11,12}$. Among these efforts, chemical recycling is viewed as an evolving solution to the plastic pollution challenge. However, because harsh conditions are usually required for chemical recycling of plastics, and low selectivity and poor product yields are generally observed ${ }^{13-15}$, especially for the chemical inert polymers such as polyethylene (PE), polypropylene (PP), and polystyrene (PS), those methods are not considered efficient enough (less than $10 \%$ plastic has been recycled in 2018) ${ }^{5}$. So, it is an urgent task to develop efficient methods for chemical recycling of plastics under gentle and energy saving conditions, which redefine plastic waste as a source of chemical feedstock, and as themselves benefits to a greener future (Fig. 1a) ${ }^{1-3}$. 


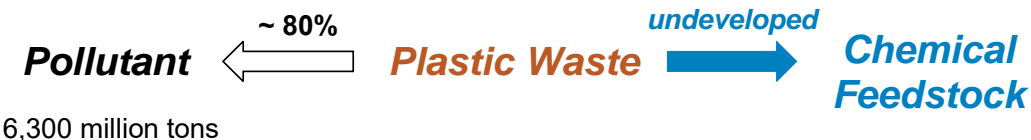

b

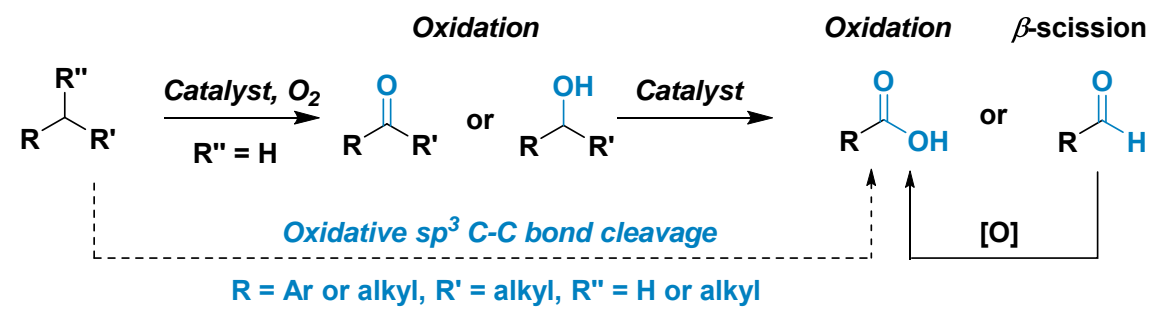

c

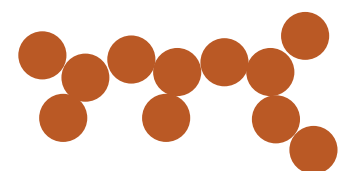

Plastic Waste

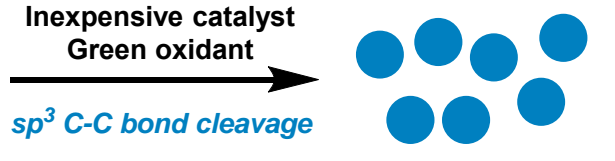

Product with commercial value

Fig. 1 | Selective degradation of plastic waste. a, Undeveloped transformation of plastic waste to chemicals. b, Design of oxidative $\mathrm{sp}^{3} \mathrm{C}-\mathrm{C}$ bond cleavage. $\mathbf{c}$, Design of selective degradation of plastic waste through oxidative $\mathrm{sp}^{3} \mathrm{C}-\mathrm{C}$ bond cleavage.

Efficient chemical recycling of plastic usually means efficient cleavage/transformation of C-C bonds, especially for the nonpolar polyolefins, which account for approximately two-thirds of the whole plastic system ${ }^{1,16}$. Because of the large dissociation energy of the $\mathrm{C}-\mathrm{C}$ single bond (around $356 \mathrm{~kJ} / \mathrm{mol}$ ), selective $\mathrm{C}-\mathrm{C}$ cleavage is always a tough challenge for chemists ${ }^{17-23}$ and hinders polymer recycle procedures $^{2,3,9,13}$. For a sustainable chemical recycling system of plastic, economical and energy saving methods to dissociate C-C bond with high efficiency are highly desired. Earth abundant metals were found to transform alkanes to alcohols and ketones under oxygen atmosphere ${ }^{24-33}$. In addition, C-C bond scission of alcohols and ketones through alkoxyl radical intermediates applying inexpensive catalysts were reported recently ${ }^{34-43}$. Though with limitations, these results illustrate us the possibility of developing an economic system for oxidative $\mathrm{C}-\mathrm{C}$ bond cleavage in an environmentally friendly way (Fig. 1b). Especially, iron may be a good catalyst candidate, which can 
perform both $\mathrm{C}-\mathrm{H}$ bond oxidation ${ }^{25,26,28}$ and oxidative $\mathrm{C}-\mathrm{C}$ bond cleavage ${ }^{43}$ through photocatalysis ${ }^{44-49}$. The two kinds of transformations may proceed in a one-pot procedure to realize the degradation of plastics through multiple $\mathrm{C}-\mathrm{H}$ bond oxidation and $\mathrm{C}-\mathrm{C}$ bond cleavage (Fig. 1c). Herein, we report an unpresented procedure for plastic depolymerization through oxidative cleavage of $\mathrm{sp}^{3} \mathrm{C}-\mathrm{C}$ bond, using iron as the catalyst under visible light in air/oxygen atmosphere. A simplified approach can be applied in the absence of solvent and additives under natural conditions, showing a significant accelerated speed of depolymerization. As far as we know, the method presented the gentlest conditions for the nonpolar polymer degradation ever reported.

\section{Results}

Initial investigation. The research begins with the study of the model substrate 1,3diphenylpropane (Table 1). A variety of conditions were tested, and iron salts were found to be effective catalysts. The reaction was firstly performed in $\mathrm{MeCN}$ under the irradiation of LED light (400 nm, see Supplementary Fig. 1-3) in the atmosphere of 1 atm of oxygen. Iron salts with different anions were investigated, many of which showed catalytic activities, presenting ketone and the targeted $\mathrm{C}-\mathrm{C}$ bond cleavage product benzoic acid as the products (entries 1-6). Benzoic acid and its additives are important chemical feedstock and widely used as food additives and precursors for industry and pharmacy. The global demand for benzoic acid is 480 kilotons in 2014 and will expand to 640 kilotons by $2024^{50}$. When $\mathrm{FeCl}_{2}$ was applied, full conversion and 90\% NMR yield (86\% isolated yield) of benzoic acid was observed in $12 \mathrm{~h}$ (entry 6). Good conversions were observed when applying other solvents, and a 71\% yield of benzoic acid was achieved when ethyl acetate or acetone was used (entries 7-9). Increasing the reaction time to $16 \mathrm{~h}$ led to an isolated yield of $88 \%$ (entry 10 ). Decreasing the catalyst loading to $2 \mathrm{~mol} \%$ resulted in full conversion of the substrate but a lower benzoic acid yield of 73\% (entry 11); while longer reaction time presented a good isolated yield of $87 \%$ (entry 12). The combination of $\mathrm{Fe}(\mathrm{OTf})_{3}$ and $\mathrm{TBACl}$ in a 1: 2 mol ratio resulted in a similar result as applying $\mathrm{FeCl}_{2}$ (entry 13). Without catalyst (entry 14) or LED light (entry 15) led to no conversion of the substrate. 
Table 1 | Optimized study of oxidative C-C bond cleavage

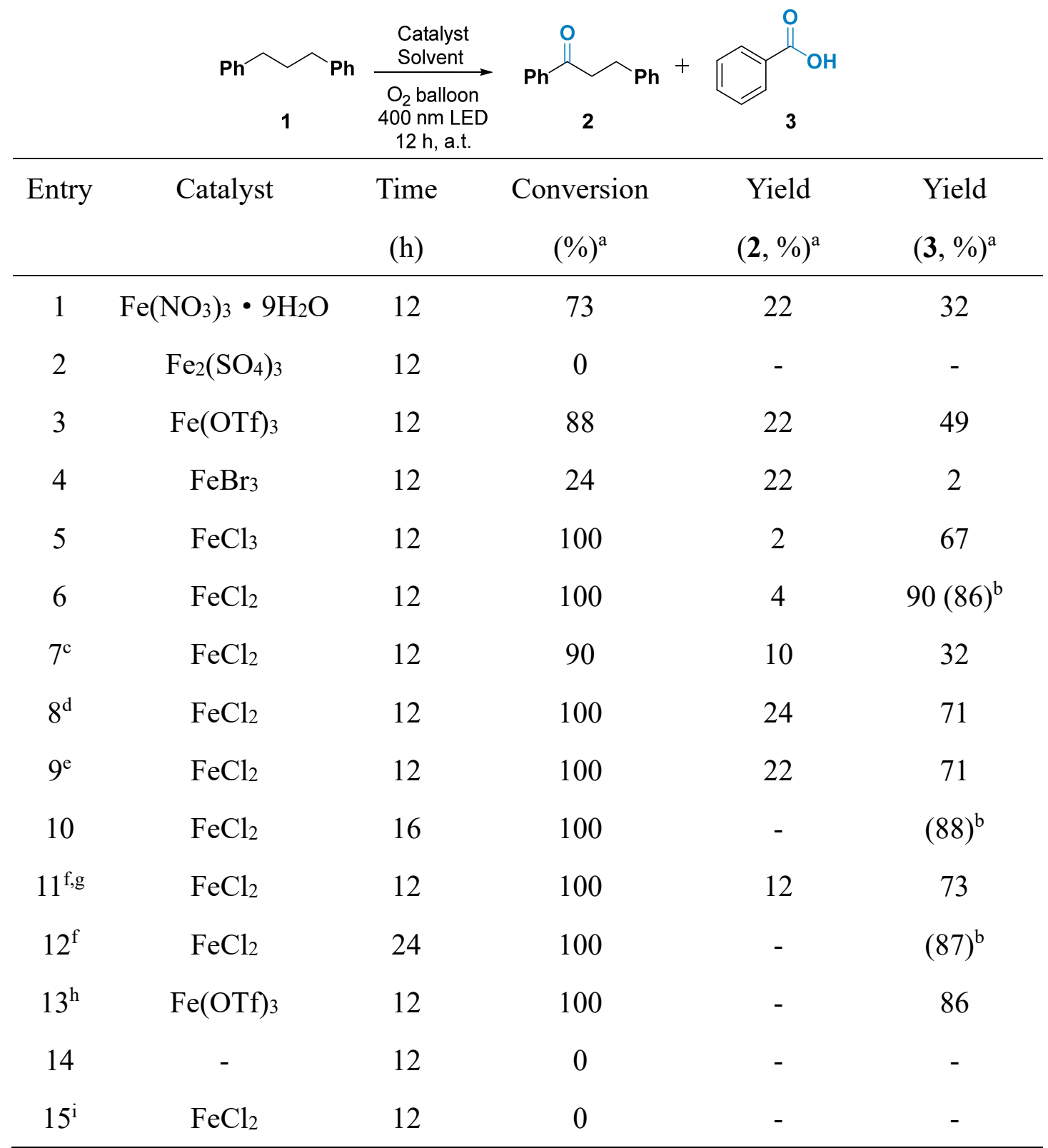

Reaction conditions: 1,3-diphenylpropane (0.1 mmol), catalyst (10 mol\%) and $\mathrm{MeCN}$ $(2 \mathrm{~mL})$ were stirred under $\mathrm{O}_{2}(1 \mathrm{~atm})$ at ambient temperature for $12 \mathrm{~h}$, irradiated by LED (400 nm, $20 \mathrm{~W})$. ${ }^{\mathrm{a}}$ Determined by ${ }^{1} \mathrm{H}$ NMR using dibromomethane as an internal standard. ${ }^{b}$ Isolated yield. ${ }^{\mathrm{c}} \mathrm{DCM}$ was used as the solvent. ${ }^{\mathrm{d}}$ Ethyl acetate was used as the solvent. ${ }^{\mathrm{e}}$ Acetone was used as the solvent. ${ }^{\mathrm{f}} 2 \mathrm{~mol} \% \mathrm{FeCl}_{2}$ was used. ${ }^{\mathrm{g}} 4 \%$ yield of 1,3-diphenylpropan-2-one was observed. ${ }^{\mathrm{h}} 20 \mathrm{~mol} \% \mathrm{TBACl}$ (tetrabutylammonium chloride) was used. ${ }^{i}$ Without LED.

Degradation of plastic. With the optimized conditions of the model substrate in hand, 
we started to study the degradation of PS. It's noteworthy that PS is usually discarded directly after use, and the relevant chemical recycling methods are overly underdeveloped ${ }^{2}$. Unfortunately, the transformation was failed at first when applying $\mathrm{MeCN}$ as the solvent, which cannot dissolve polystyrene. Plenty of solvents were tested, and dichloromethane (DCM) and acetone showed good solubility for PS. Surprisingly, the degradation of dissolved PS easily happened under the gentle optimized conditions for the model substrate. The mixed solvent with a volume ratio of 3: 2 (DCM: MeCN) led to good performance. When applying $2 \mathrm{~mol}^{\circ} \mathrm{FeCl}_{2}$ as the catalyst, depolymerization was quickly preceded with the formation of carbonyl bonds (entries 1-5, Fig. 2a), and the polymer molecular weight (Mw) was dropped from $145 \mathrm{~kg} / \mathrm{mol}$ to $5 \mathrm{~kg} / \mathrm{mol}$ in just $1 \mathrm{~h}$ (entry 1$)$. Full conversion of oligomer $(\mathrm{Mw}>1 \mathrm{~kg} / \mathrm{mol})$ was achieved in $6 \mathrm{~h}$ (entry 6, Fig. 2b). A longer time was required for better reaction selectivity, and $63 \%$ isolated yield of benzoic acid was produced after $66 \mathrm{~h}$ (entries 79). Side products in small amounts, such as benzaldehyde and acetophenone, were observed by GC-MS. No oligomer or large molecule was observed. When acetone was applied as the solvent, a yield of $61 \%$ was presented (entry 10). Using $\mathrm{FeCl}_{3}$ as a catalyst led to a similar yield of $62 \%$ (entry 11 ). Different combinations of iron salts and TBACl were also investigated, yields between $49 \%$ to $58 \%$ were observed (entries 12-15), and $\mathrm{Fe}_{2}\left(\mathrm{SO}_{4}\right)_{3}$ resulted in the best NMR yield of $66 \%$ (entry 16). Interestingly, when the reactions were performed under air in different solvents, good results were also achieved with higher $\mathrm{FeCl}_{2}$ loading of $10 \mathrm{~mol} \%$ (entries 17, 18). Decreasing the catalyst loading to $0.1 \mathrm{~mol} \%$ didn't reduce the conversion, resulting in a $26 \%$ isolated yield of benzoic acid as the product. This result highlights the efficiency of the catalysis system for PS degradation.

Table 2 | Degradation study of polystyrene through oxidative $\mathrm{C}-\mathrm{C}$ bond cleavage

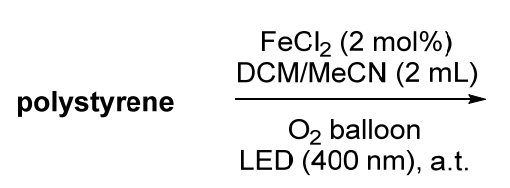

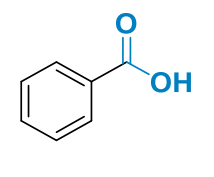

\begin{tabular}{lllll}
\hline Entry & Catalyst & Time $(\mathrm{h})$ & Conversion $(\%)^{\mathrm{a}}$ & Yield $_{(\%)^{\mathrm{b}}}$ \\
\hline
\end{tabular}




\begin{tabular}{|c|c|c|c|c|}
\hline 1 & $\mathrm{FeCl}_{2}$ & 1 & 14 & 0 \\
\hline 2 & $\mathrm{FeCl}_{2}$ & 2 & 24 & 0 \\
\hline 3 & $\mathrm{FeCl}_{2}$ & 3 & 41 & 0 \\
\hline 4 & $\mathrm{FeCl}_{2}$ & 4 & 60 & 0 \\
\hline 5 & $\mathrm{FeCl}_{2}$ & 5 & 95 & 0 \\
\hline 6 & $\mathrm{FeCl}_{2}$ & 6 & 100 & 4 \\
\hline 7 & $\mathrm{FeCl}_{2}$ & 12 & 100 & 29 \\
\hline 8 & $\mathrm{FeCl}_{2}$ & 48 & 100 & 55 \\
\hline 9 & $\mathrm{FeCl}_{2}$ & 66 & 100 & $65(63)^{\mathrm{c}}$ \\
\hline $10^{\mathrm{d}}$ & $\mathrm{FeCl}_{2}$ & 66 & 100 & $61(59)^{\mathrm{c}}$ \\
\hline 11 & $\mathrm{FeCl}_{3}$ & 66 & 100 & 62 \\
\hline $12^{\mathrm{e}}$ & $\mathrm{Fe}(\mathrm{OAc})_{2} \mathrm{OH}$ & 66 & 100 & 49 \\
\hline $13^{\mathrm{e}}$ & $\mathrm{Fe}(\mathrm{acac})_{3}$ & 66 & 100 & 56 \\
\hline $14^{\mathrm{e}}$ & $\mathrm{Fe}(\mathrm{OTf})_{3}$ & 66 & 100 & 57 \\
\hline $15^{\mathrm{e}}$ & $\mathrm{Fe}\left(\mathrm{NO}_{3}\right)_{3} \cdot 9 \mathrm{H}_{2} \mathrm{O}$ & 66 & 100 & 58 \\
\hline $16^{\mathrm{e}}$ & $\mathrm{Fe}_{2}\left(\mathrm{SO}_{4}\right)_{3}$ & 66 & 100 & 66 \\
\hline $17^{\mathrm{f}}$ & $\mathrm{FeCl}_{2}$ & 66 & 100 & 62 \\
\hline $18^{\mathrm{f}}$ & $\mathrm{FeCl}_{2}$ & 66 & 100 & $63^{\mathrm{d}}$ \\
\hline $19^{\mathrm{g}}$ & $\mathrm{FeCl}_{2}$ & 66 & 100 & $(26)^{c}$ \\
\hline
\end{tabular}

Reaction conditions: polystyrene ( $\mathrm{Mw}$ is around $145 \mathrm{~kg} / \mathrm{mol}, 0.5 \mathrm{mmol}$ based on $\left.\mathrm{C}_{8} \mathrm{H}_{8}\right)$, catalyst (2 mol\%), DCM/MeCN (v: $\left.\mathrm{v}=3: 2,2 \mathrm{~mL}\right)$ were stirred under $\mathrm{O}_{2}(1$ atm) at ambient temperature (a.t.) for $12 \mathrm{~h}$, irradiated by LED (400 nm, $20 \mathrm{~W})$. ${ }^{\mathrm{a} B a s e d}$ on the isolated yield of oligomers. ${ }^{b}$ Yield of benzoic acid determined by ${ }^{1} \mathrm{H}$ NMR using dibromomethane as an internal standard. ${ }^{\mathrm{c}}$ Isolated yield. ${ }^{\mathrm{d}}$ Acetone was used as solvent. ${ }^{\mathrm{e}} 4 \mathrm{~mol} \% \mathrm{TBACl}$ (tetrabutylammonium chloride) was used. ${ }^{\mathrm{f}} \mathrm{Air}$ was used instead of oxygen, $10 \mathrm{~mol} \% \mathrm{FeCl}_{2}$ was applied. ${ }^{\mathrm{g}} 1$,2-dicloroethane was used as solvent, $0.1 \mathrm{~mol} \% \mathrm{FeCl}_{2}$ was applied. 

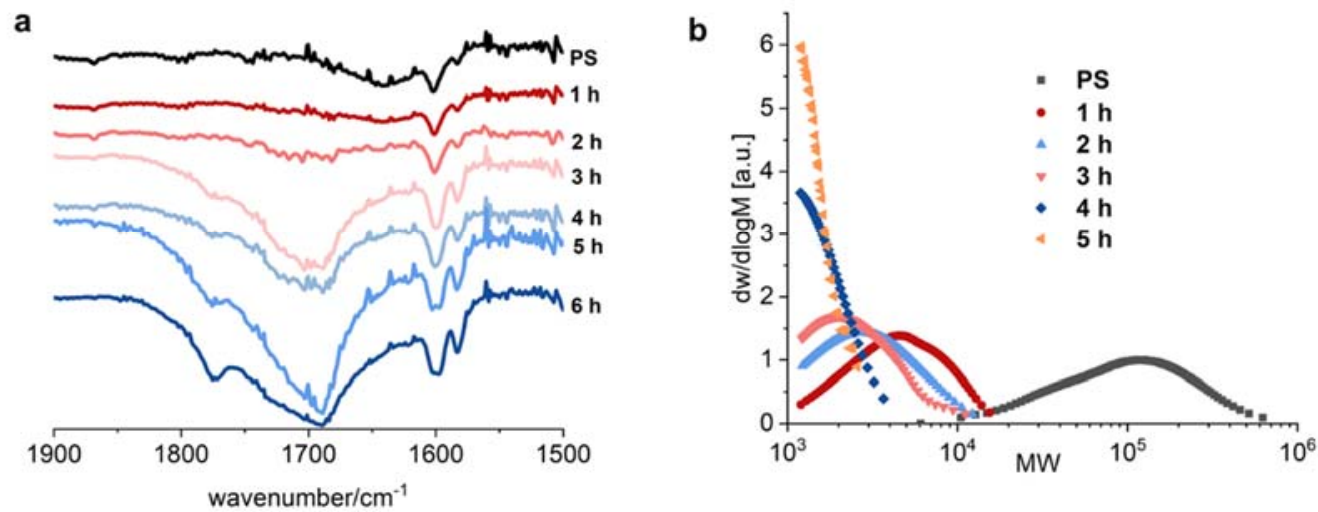

Fig. 2 | Spectra of degradation of PS in mixed DCM/MeCN (3: 2). a, FT-IR spectra of PS degradation reaction in 1-6 h. b, GPC traces of PS degradation reaction in 1-5 h.

Later, the simple system was applied to degrade different kinds of plastics (Table 3). $\mathrm{FeCl}_{2}$ was applied as the catalyst, and two solvent systems ( $\mathrm{DCM} / \mathrm{MeCN}$, acetone) were investigated. Generally, full conversions were observed. PS particles with different molecular weight were smoothly depolymerized, producing benzoic acid selectively in moderate isolated yields in the mixed solvent DCM/MeCN. PS with lower molecular weight resulted in better isolated yield of benzoic acid when acetone was applied as the solvent (entry 1 vs. 2). Syndiotactic PS and atactic PS were also successfully transformed to benzoic acid in DCM/MeCN, with $68 \%$ and $67 \%$ isolated yield, respectively (entries 3 and 4). Different copolymers of styrene were also tested under lower catalyst loading of $1 \mathrm{~mol} \%$ or $0.8 \mathrm{~mol} \%$ (entries 5-8), styrene-allyl alcohol copolymer presented a similar reactivity and selectivity as PS (entry 5). Poly(styreneco-acrylonitrile) (entry 6), ABS (acrylonitrile butadiene styrene polymer) (entry 7) and styrene-maleic anhydride copolymer (entry 8) presented lower yields of benzoic acid with full conversion. Delightfully, commercial plastic waste produced from PS (see Supplementary Fig. 4), such as PS foam board (entry 9) and PS cup (entry 10), can also be depolymerized in full conversion, yielding a similar amount of benzoic acid as compared to pure PS. The results are attractive since commercial plastics usually containing additives such as antioxidants to extend the plastic life, which don't decrease the catalytic activity. 
Table 3 | Depolymerization of different polymers through oxidative C-C bond cleavage

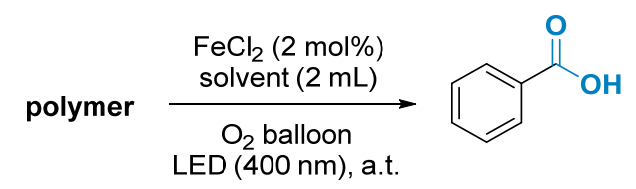

\begin{tabular}{llccc}
\hline Entry & Substrate (Mw, kg/mol) & $\begin{array}{c}\text { Conversion } \\
(\%)^{\mathrm{a}}\end{array}$ & $\begin{array}{c}\text { Yield } \\
(\%)^{\mathrm{b}, \mathrm{c}}\end{array}$ & $\begin{array}{c}\text { Yield } \\
(\%)^{\mathrm{b}, \mathrm{d}}\end{array}$ \\
\hline 1 & PS particle (35) & 100 & 56 & 62 \\
2 & PS particle (350) & 100 & 56 & $51^{\mathrm{e}}$ \\
3 & syndiotactic PS (222) & 100 & 68 & 61 \\
4 & atactic PS (202) & 100 & 67 & 66 \\
$5^{\mathrm{f}, \mathrm{g}}$ & Styrene-allyl alcohol copolymer (4) & 100 & 56 & 57 \\
$6^{\mathrm{h}}$ & Poly(styrene-co-acrylonitrile) (106) & 100 & 33 & 23 \\
$7^{\mathrm{g}, \mathrm{i}}$ & ABS (124) & 100 & 31 & $3^{\mathrm{j}}$ \\
$8^{\mathrm{g}, \mathrm{k}}$ & Styrene maleic anhydride copolymer & 100 & 44 & 43 \\
& (74) & & & 61 \\
9 & Waste PS foam board (222) & 100 & 63 & 65 \\
\hline 10 & Waste PS cup (166) & 100 & 63 \\
\hline
\end{tabular}

Reaction conditions: polymer ( $0.5 \mathrm{mmol}$ based on monomer), $\mathrm{FeCl}_{2}(2 \mathrm{~mol} \%)$, and solvent $(2 \mathrm{~mL})$ were stirred under $\mathrm{O}_{2}(1 \mathrm{~atm})$ at ambient temperature (a.t.) for $12 \mathrm{~h}$,

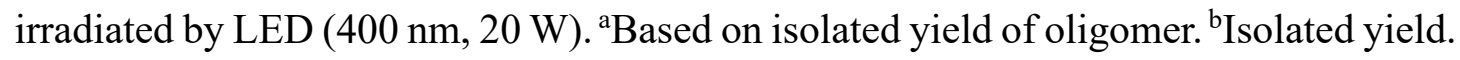
${ }^{\mathrm{c}} \mathrm{DCM} / \mathrm{MeCN}$ (3: 2) was used as the solvent. ${ }^{\mathrm{d}}$ Acetone was used as the solvent. ${ }^{\mathrm{e}} \mathrm{The}$ conversion was 62\%. ${ }^{\mathrm{f}} 0.96 \mathrm{mmol}$ styrene-allyl alcohol copolymer (based on $\left.\left(\mathrm{C}_{8} \mathrm{H}_{8}\right)_{0.57}\left(\mathrm{C}_{3} \mathrm{H}_{6} \mathrm{O}\right)_{0.43}\right)$ was used. ${ }^{\mathrm{g}} 1 \mathrm{~mol} \% \mathrm{FeCl}_{2}$ was applied. ${ }^{\mathrm{h}} 1.26 \mathrm{mmol}$ Poly(styreneco-acrylonitrile) (based on $\left.\left(\mathrm{C}_{8} \mathrm{H}_{8}\right)_{0.6}\left(\mathrm{C}_{3} \mathrm{H}_{3} \mathrm{~N}\right)_{0.4}\right)$ was used. $0.8 \mathrm{~mol} \% \mathrm{FeCl}_{2}$ was applied. i1 mmol acrylonitrile butadiene styrene polymer (ABS, based on $\left.\left(\mathrm{C}_{8} \mathrm{H}_{8}\right)_{0.5}\left(\mathrm{C}_{4} \mathrm{H}_{6}\right)_{0.25}\left(\mathrm{C}_{3} \mathrm{H}_{3} \mathrm{~N}\right)_{0.25}\right)$ was used. ${ }^{\mathrm{j}}$ The conversion was $18 \% .{ }^{\mathrm{k}} 1 \mathrm{mmol}$ of styrene maleic anhydride copolymer (based on $\left.\left(\mathrm{C}_{8} \mathrm{H}_{8}\right)_{0.43}\left(\mathrm{C}_{4} \mathrm{H}_{2} \mathrm{O}_{3}\right)_{0.57}\right)$ was used. 
Degradation of plastic in the absence of solvent in air. Furthermore, a more simplified procedure was tried to check the degradation ability of PS in the absence of solvent in the air (Table 4). PS was dissolved and mixed with $\mathrm{FeCl}_{2}$, the solvent was then evaporated and the resulting film was irradiated with $400 \mathrm{~nm}$ LED or sunlight in the air (Supplementary Fig. 5). Interestingly, the samples with $\mathrm{FeCl}_{2}$ showed significantly faster degradation speed ( $\mathrm{Mw}_{1}$, entries 1-3) than the samples without (Mw2, entries 1-3) under LED (Fig. 3a-d). The depolymerization also happened observably under natural conditions for the iron-containing samples (Mw3, entries 1-4), while no degradation was observed for iron-free samples (Mw4, entries 1-4) (Fig. 3e-f). In addition, for samples with iron, signals of carbonyl groups, which can further improve the degradation of the polymer, were detected by IR (Fig. 3c,g). Iron is one of the most abundant and environmentally friendly metals in the earth. These observations highlight the potential application of iron salts as additives for disposable plastics and plastics used for a short period, which don't change the properties when use and can be degraded fast by simple treatment or just discarded in the natural environment.

Table 4 | Degradation of polystyrene in solid state

\begin{tabular}{cccccc}
\hline Entry & Time (day) & $\mathrm{Mw}_{1}{ }^{\mathrm{b}}$ & $\mathrm{Mw}_{2}{ }^{\mathrm{c}}$ & $\mathrm{Mw}_{3}^{\mathrm{d}, \mathrm{e}}$ & $\mathrm{Mw}_{4}{ }^{\mathrm{e}, \mathrm{f}}$ \\
\hline 1 & 1 & 55 & 112 & 129 & 144 \\
2 & 3 & 50 & 95 & 105 & 147 \\
3 & 6 & 43 & 89 & 101 & 148 \\
4 & 12 & $-\mathrm{g}$ & $-\mathrm{g}$ & 97 & 144 \\
\hline
\end{tabular}

${ }^{\text {a}}$ Reaction conditions: polystyrene, $\mathrm{FeCl}_{2}$ (2 mol\%), DCM: $\mathrm{MeCN}(3: 2)$ were mixed and well dissolved. Then the solution was separated and transferred to different vials. After the solvent was removed, the vials were irradiated by $400 \mathrm{~nm}$ LED (50 W) or natural sunlight under air. The unit of $\mathrm{Mw}$ is $\mathrm{kg} / \mathrm{mol}$. GPC was applied to measure Mw. ${ }^{b} \mathrm{LED}$ was used. ${ }^{\mathrm{c}} \mathrm{LED}$ was used without $\mathrm{FeCl}_{2}$. ${ }^{\mathrm{d}}$ Under natural sunlight. ${ }^{\mathrm{e}}$ The time of daylength is around $13 \mathrm{~h}$, and the latitude is 23 degrees, the temperature is between 23-31 ${ }^{\circ} \mathrm{C}$. fUnder natural sunlight without $\mathrm{FeCl}_{2}$. ${ }^{\mathrm{g}} \mathrm{Not}$ tested. 

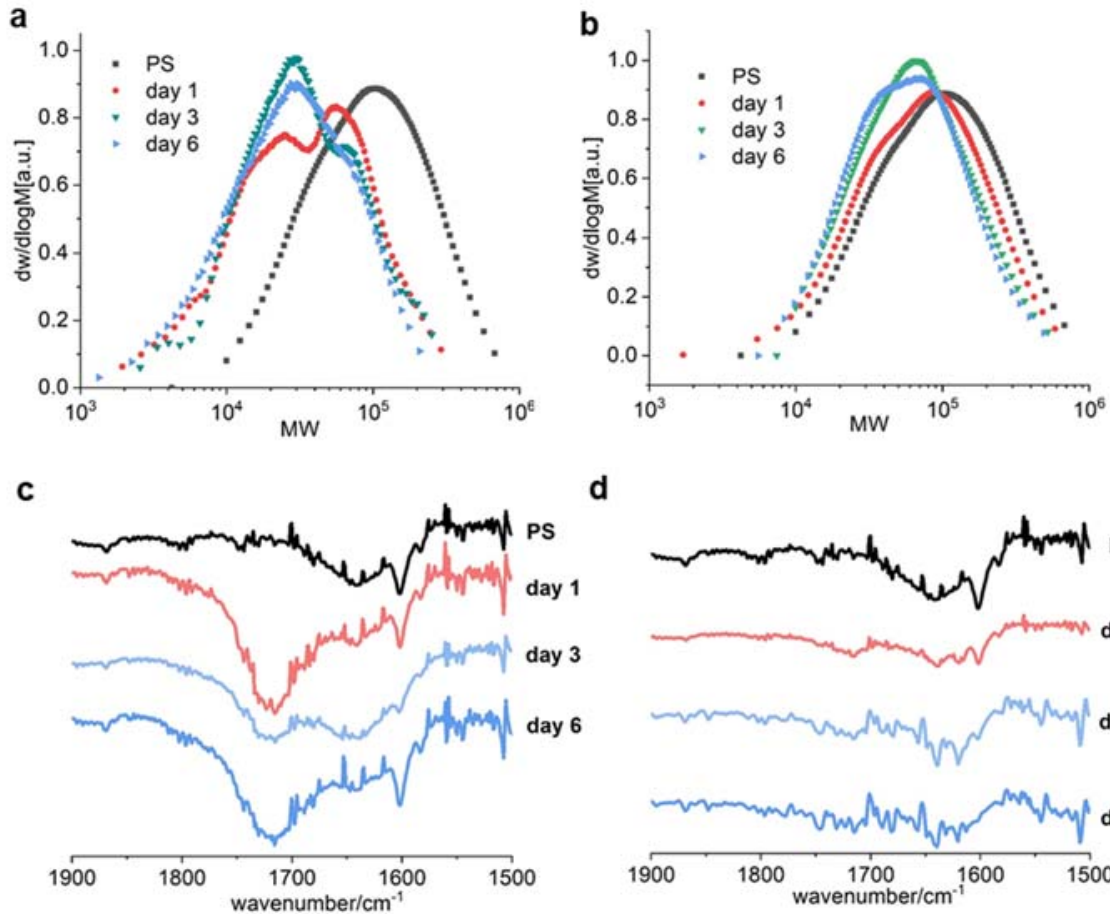

d

e
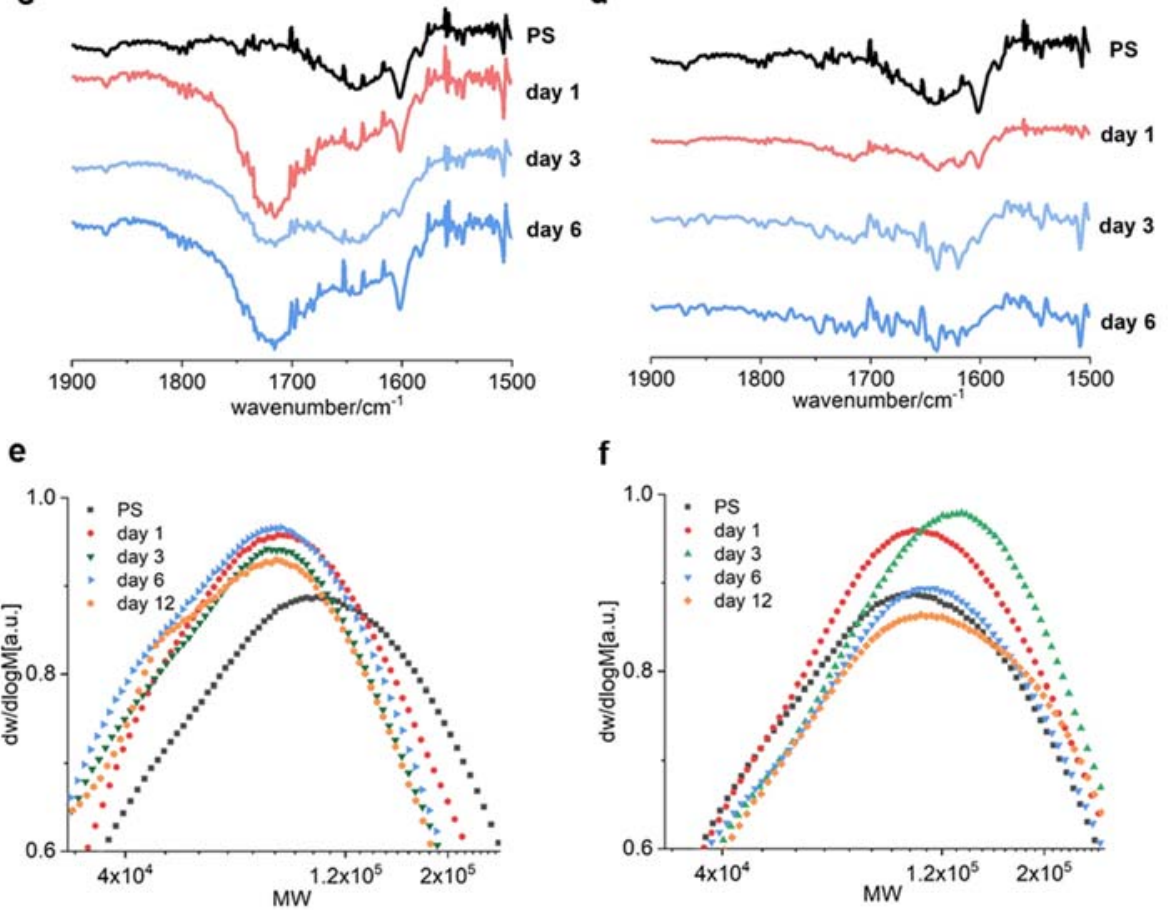

f
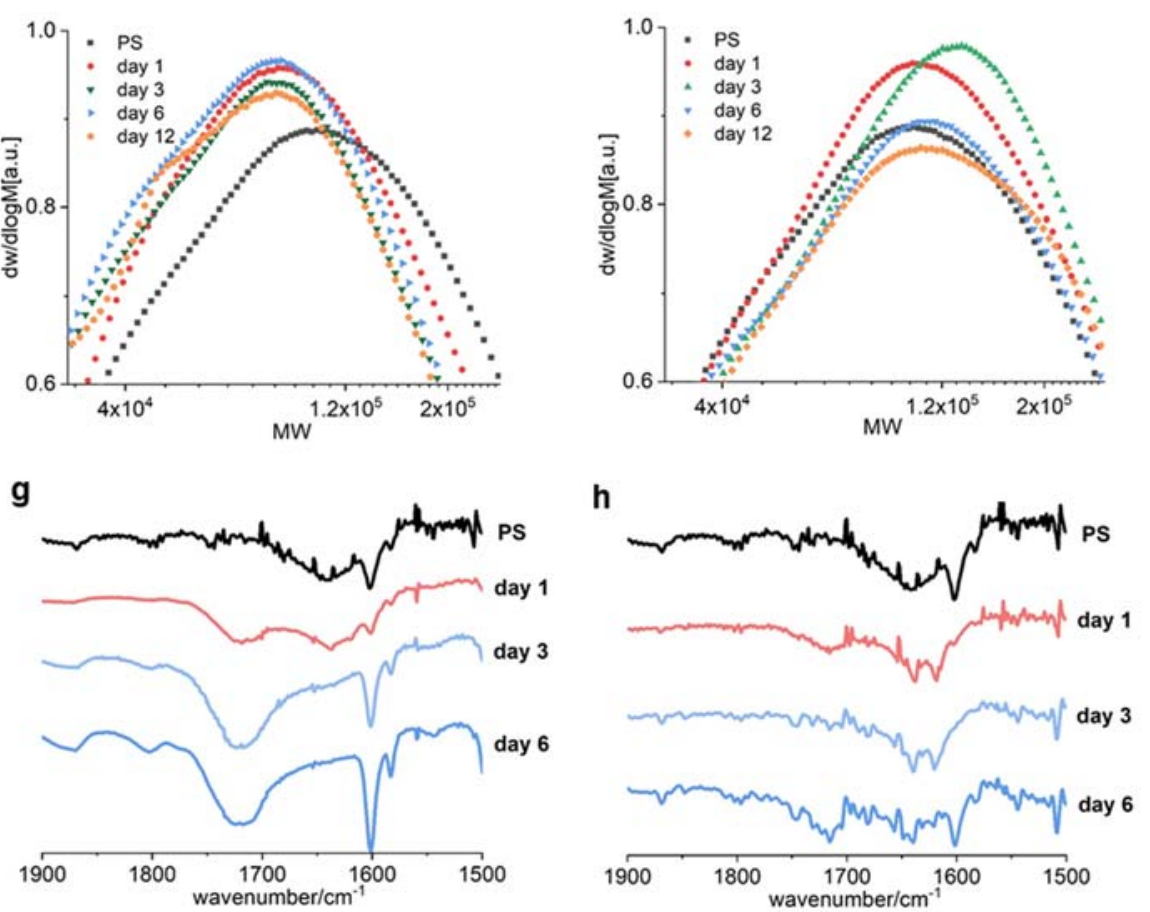

Fig. 3 | Spectra of degradation of PS in solid state. a, b, GPC traces of PS containing (a) or without (b) $2 \mathrm{~mol} \% \mathrm{FeCl}_{2}$ after irradiation with $400 \mathrm{~nm} \mathrm{LED.} \mathrm{c,} \mathrm{d,} \mathrm{FT-IR} \mathrm{spectra}$ of PS containing (c) or without (d) $2 \mathrm{~mol} \% \mathrm{FeCl}_{2}$ after irradiation with $400 \mathrm{~nm}$ LED. e, f, GPC traces of PS containing (e) or without (f) $2 \mathrm{~mol} \% \mathrm{FeCl}_{2}$ after irradiation with sunlight outdoors (see Supplementary Fig. 6,7 for detailed spectra of GPC traces). g, h, FT-IR spectra of PS containing (g) or without (h) $2 \mathrm{~mol} \% \mathrm{FeCl}_{2}$ after irradiation with 
sunlight outdoors.
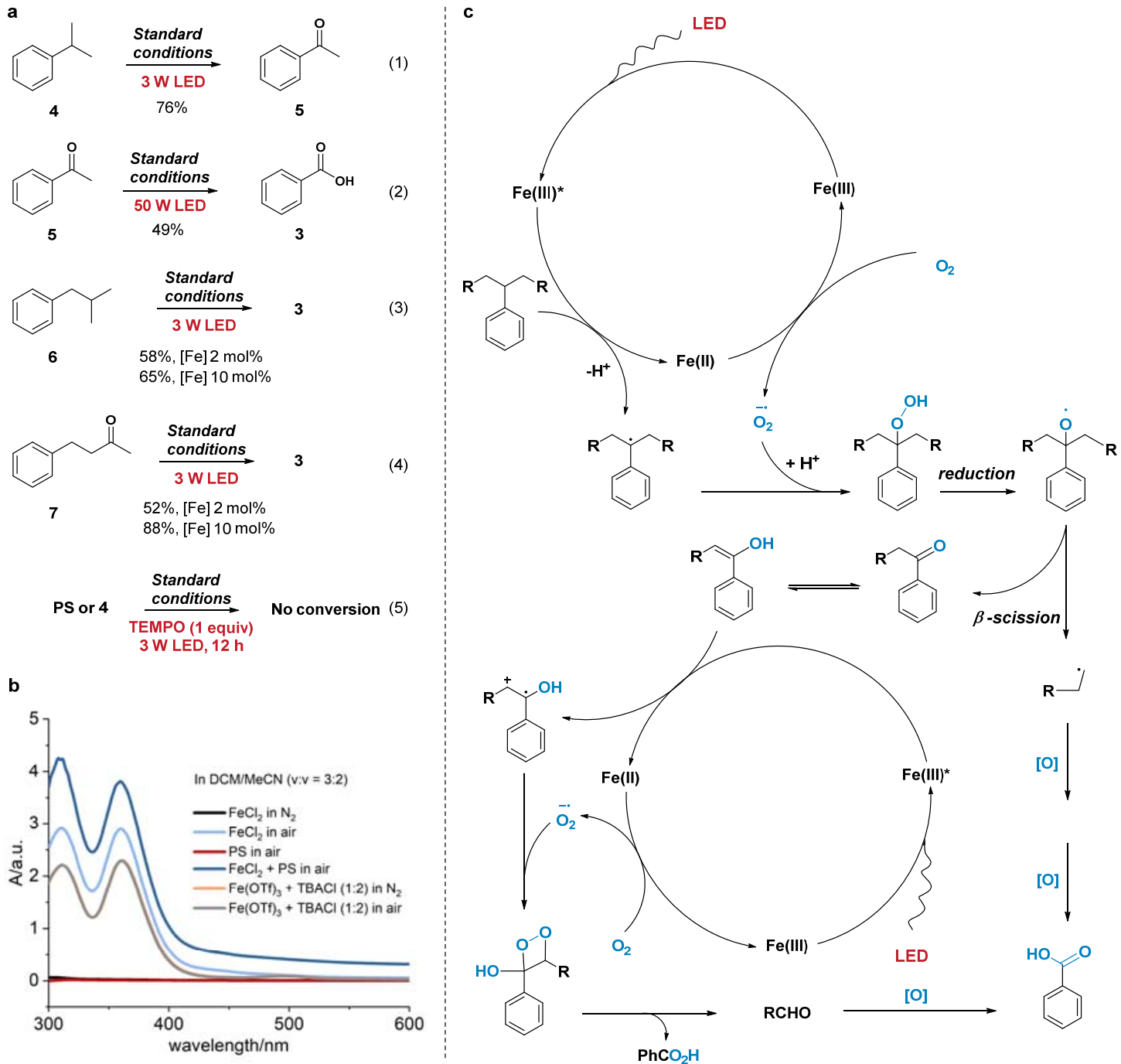

Fig. 4 | Mechanism study. a, Substrate performance under LEDs with different power. Standard conditions: substrate (0.1 or $0.2 \mathrm{mmol}), \mathrm{FeCl}_{2}(2 \mathrm{~mol} \%)$ and $\mathrm{MeCN}(2 \mathrm{~mL})$ were stirred under $\mathrm{O}_{2}(1 \mathrm{~atm})$ at ambient temperature for $24 \mathrm{~h}$, irradiated by LED (400 nm) (see Supplementary Fig. 8 for detailed condition). Acetophenone yield was measured by GC-MS using mesitylene as an internal standard. Other yields are isolated yields. b, UV-vis spectra under $\mathrm{N}_{2}$ or air. DCM/MeCN (v: v = 3: 2) was used as the solvent (in the case of using acetone as the solvent, see Supplementary Fig. 9). Concentration: $5 \times 10^{-4} \mathrm{~mol} / \mathrm{L}[\mathrm{Fe}], 2.5 \times 10^{-2} \mathrm{~mol} / \mathrm{L}$ PS. c, Proposed mechanism.

Mechanistic investigation. To study the mechanism, we set some reactions using 
simplely substituted arenes as substrates (Fig. 4a, Supplementary Fig. 8). For reactant with a tertiary benzylic carbon, such as isopropyl benzene (4), full conversion and $76 \%$ GC-MS yield of acetophenone (5) was easily achieved under the irradiation of $3 \mathrm{~W}$ LED. However, only trace benzoic acid was observed. The higher power of LED was found to be an essential factor for further oxidation of acetophenone. When $50 \mathrm{~W}$ LED was used, benzoic acid was generated from both isopropyl benzene (see Supplementary Fig. 8) and acetophenone. In addition, isobutylbenzene (6) and 4-phenylbutan-2-one (7) were transformed to benzoic acid smoothly when applying $3 \mathrm{~W}$ LED. Based on the optimized study and our observation of the above reactions, ketone is surely a key intermediate product of the oxidative $\mathrm{C}-\mathrm{C}$ bond cleavage procedure. Both $\mathrm{sp}^{3} \mathrm{C}-\mathrm{C}$ bond oxidative cleavage of tertiary benzylic carbon (such as 4 ) and $\mathrm{C}-\mathrm{H}$ oxidation of benzylic $\mathrm{C}-\mathrm{H}$ bonds to produce ketones can be easily achieved under the standard conditions. However, oxidation of acetophenone is much harder than the other ketone intermediates (see Supplementary Fig. 8). One major difference between these ketones that affect the reactivity should be the ratio of their enol forms, which may be oxidized easily under the reaction conditions ${ }^{51}$. From the reaction condition studies and the UV-vis spectra (Fig. 4b and Supplementary Fig. 9), Fe (III) is believed to be the real photocatalyst, which can be oxidized from Fe (II) simply under oxygen atmosphere. Based on the above observations and the relevant mechanism studies reported ${ }^{28,43,51-55}$, the proposed mechanism is shown in Fig. 4c. The irradiated Fe(III) oxidizes the benzylic C-H bond, generating the corresponding radical, which is then captured by superoxide radical and proton to produce the hydroperoxide ${ }^{28,52,53}$. Reduction of the hydroperoxide by Fe(II) and the following beta-scission leads to the formation of the ketone. The enol form of ketone then reacts with oxygen to generate a four-membered ring, which decomposes to yield benzoic acid as the product ${ }^{53-55}$. Benzaldehyde can transform to benzoic acid smoothly under the reaction conditions (see Supplementary Fig. 8). Phenyl alkyl radicals can also produce benzoic acid through similar multiple oxidation procedures.

\section{Conclusion}

In conclusion, we developed a procedure to enable efficient oxidative multiple $\mathrm{sp}^{3}$ $\mathrm{C}-\mathrm{C}$ bond cleavage, which can be applied to depolymerize plastics containing styrene 
selectively, generating commercial valuable benzoic acid as the product. The system is also inexpensive and environmentally friendly. Performed under visible light, the method uses iron, one of the most abundant metals on the earth, as the catalyst, oxygen or air as the oxidizing agent. Many commercial plastics, besides polystyrene waste, show outstanding conversions and very high selectivity of benzoic acid production. In addition, the system can be applied to degrade plastics under solvent free conditions, even in natural conditions, suggesting the potential application of iron salts as additives for degradable plastics.

\section{Methods}

General. All reagents were obtained from commercial vendors and used as received unless otherwise noted. Acetonitrile and dichloromethane were dried using calcium hydride. Flash column chromatography was performed using silica gel (300-400 mesh). ${ }^{1} \mathrm{H}$ NMR spectra were recorded at $400 \mathrm{MHz}$, using a Bruker AVANCE III 400 (400 $\mathrm{MHz}$ ) spectrometer. Measurements were done at ambient temperature. Fouriertransform infrared (FT-IR) spectra were obtained on an interferometer of IRAffinity1S. DCM solution of the sample was dropped on the surface of a $\mathrm{KBr}$ plate and dried. Then the IR spectrum was collected. UV-vis spectra were recorded on a HORIBA Duetta compact spectrofluorometer. The cuvette used was a standard $1 \mathrm{~cm}$ width quartz cuvette. Gel-permeation chromatography (GPC) was performed on a Waters Breeze 2 GPC equipped with a refractive index detector at $35{ }^{\circ} \mathrm{C}$. Samples were run in tetrahydrofuran with a speed of $1.0 \mathrm{~mL} / \mathrm{min}$. GC-MS data were detected on an Agilent 8890-5977B gas chromatography-mass spectrometry.

General procedure for transformation of arene substrates. A $25 \mathrm{~mL}$ Schlenk tube with screw-cap was added $100 \mu \mathrm{L} \mathrm{FeCl}_{2}$ solution ( $0.1 \mathrm{~mol} / \mathrm{L}$ dissolved in acetonitrile), aromatic substrate $(0.1 \mathrm{mmol})$, and $1.9 \mathrm{~mL}$ acetonitrile. The tube was blown with oxygen for several seconds and maintained in oxygen atmosphere by adding an oxygen balloon. Then the Schlenk tube was irradiated with $400 \mathrm{~nm}$ light emitting diode (LED, $20 \mathrm{~W}$ ) and stirred at $500 \mathrm{rpm}$ for $12 \mathrm{~h}$. Subsequently, the solvent was evaporated under reduced pressure and the residue was purified by flash chromatography using petroleum ether and dichloromethane as the eluent. 
General procedure for selective degradation of plastics in DCM/MeCN. A $25 \mathrm{~mL}$ Schlenk tube with screw-cap was added $800 \mu \mathrm{L} \mathrm{FeCl}_{2}$ solution $(0.0125 \mathrm{~mol} / \mathrm{L}$ dissolved in acetonitrile), polystyrene $(0.5 \mathrm{mmol})$, and $1.2 \mathrm{~mL}$ dichloromethane. The tube was blown with oxygen for several seconds and maintained in oxygen atmosphere by adding an oxygen balloon. Then the Schlenk tube was irradiated by $400 \mathrm{~nm}$ LED (20 W) and stirred at $500 \mathrm{rpm}$ for $66 \mathrm{~h}$. Subsequently, $15 \mathrm{~mL} \mathrm{MeOH}$ was added, the solution was filtered and the resulting residue was dried and weighed to calculate the conversion of polystyrene. Then, the filtrate was evaporated under reduced pressure and the residue was purified by flash chromatography using petroleum ether and dichloromethane as the eluent.

General procedure for selective degradation of plastics in acetone. A $25 \mathrm{~mL}$ Schlenk tube with screw-cap was added $1 \mathrm{~mL} \mathrm{FeCl}_{2}$ solution $(0.01 \mathrm{~mol} / \mathrm{L}$ dissolved in acetone), polystyrene $(0.5 \mathrm{mmol})$, and $1 \mathrm{~mL}$ acetone. The tube was blown with oxygen for several seconds and maintained in oxygen atmosphere by adding an oxygen balloon. Then the Schlenk tube was irradiated by $400 \mathrm{~nm}$ LED $(20 \mathrm{~W})$ and stirred at $500 \mathrm{rpm}$ for $66 \mathrm{~h}$. Subsequently, $15 \mathrm{~mL} \mathrm{MeOH}$ was added, the solution was filtered and the resulting residue was dried and weighed to calculate the conversion of polystyrene. Then, the filtrate was evaporated under reduced pressure and the residue was purified by flash chromatography using petroleum ether and dichloromethane as the eluent.

Procedure of degradation of PS using low catalyst loading. A $25 \mathrm{~mL}$ Schlenk tube with screw-cap was added $40 \mu \mathrm{L} \mathrm{FeCl}_{2}$ solvent (0.0125 M dissolved in acetonitrile). Acetonitrile was evaporated under reduced pressure, and then polystyrene $(0.5 \mathrm{mmol})$ and dichloroethane $(2 \mathrm{~mL})$ were added. The tube was blown with oxygen for several seconds and maintained in oxygen atmosphere by adding an oxygen balloon. Then the Schlenk tube was irradiated by $400 \mathrm{~nm}$ LED $(20 \mathrm{~W})$ and stirred at $500 \mathrm{rpm}$ for $66 \mathrm{~h}$. Subsequently, $15 \mathrm{~mL} \mathrm{MeOH}$ was added, the solution was filtered and the resulting residue was dried and weighed to calculate the conversion of polystyrene. Then, the filtrate was evaporated under reduced pressure and the residue was purified by flash chromatography using petroleum ether and dichloromethane as the eluent.

General procedure for solvent free experiment. A $20 \mathrm{~mL}$ vial was added $1.6 \mathrm{~mL}$ 
$\mathrm{FeCl}_{2}$ solvent $(0.0125 \mathrm{~mol} / \mathrm{L}$ dissolved in acetonitrile $)$, polystyrene $(0.5 \mathrm{mmol})$, and 2.4 $\mathrm{mL}$ dichloromethane. The vial was then shaken violently to get a homogeneous solution. Then $80 \mu \mathrm{L}$ solution was added into a $4 \mathrm{~mL}$ headspace bottle. The solvent was removed and the bottle was irradiated by $400 \mathrm{~nm}$ LED $(20 \mathrm{~W})$ or sunlight. The FT-IR spectra and gel-permeation chromatography (GPC) were determined for different samples. At the same time, the control experiments without $\mathrm{FeCl}_{2}$ were carried out.

\section{Data availability}

The data supporting the findings of this study are available within the paper and its Supplementary Information.

\section{References}

1. Science to Enable Sustainable Plastics. A White Paper from the 8th Chemical Sciences and Society Summit (CS3); available at go.nature.com/3pfImyw (2020).

2. Worch, J. C. \& Dove, A. P. 100th anniversary of macromolecular science viewpoint: toward catalytic chemical recycling of waste (and future) plastics. ACS Macro Lett. 9, 1494-1506 (2020).

3. Fagnani, D. E., Tami, J. L., Copley, G., Clemons, M. N., Getzler, Y. D. Y. L. \& McNeil, A. J. 100th anniversary of macromolecular science viewpoint: redefining sustainable polymers. ACS Macro Lett. 10, 41-53 (2021).

4. Scholten, P. B. V., Jie, C. \& Mathers, R. T. Polymers for a sustainable future. Macromol. Rapid Commun. 42, 2000745 (2021).

5. Geyer, R., Jambeck, J. R. \& Law, K. L. Production, use, and fate of all plastics ever made. Sci. Adv. 3, e1700782 (2017).

6. Mathers, R. T. How well can renewable resources mimic commodity monomers and polymers? J. Polym. Sci., Part A: Polym. Chem. 50, 1-15 (2012).

7. Parkatzidis, K., Wang, H. S., Truong, N. P. \& Anastasaki, A. Recent developments and future challenges in controlled radical polymerization: a 2020 update. Chem 6, 1575-1588 (2020).

8. Bioplastics Market Data 2019. European Bioplastics; european-bioplastics.org/market/

9. Häußler, M., Eck, M., Rothauer, D. \& Mecking, S. Closed-loop recycling of 
polyethylene-like materials. Nature 590, 423-427 (2021).

10. Rapra, S. Handbook of Plastics Recycling: Shrewsbury, UK (2002)

11. Ragaert, K., Delva, L. \& Van Geem, K. Mechanical and chemical recycling of solid plastic waste. Waste Manag. 69, 24-58 (2017).

12. Saito, K., Jehanno, C., Meabe, L., Olmedo-Martínez, J. L., Mecerreyes, D., Fukushima, K. \& Sardon, H. From plastic waste to polymer electrolytes for batteries through chemical upcycling of polycarbonate. J. Mater. Chem. A 8, 13921-13926 (2020).

13. Lopez, G., Artetxe, M., Amutio, M., Bilbao, J. \& Olazar, M. Thermochemical routes for the valorization of waste polyolefinic plastics to produce fuels and chemicals. A review. Renew. Sustain. Energy Rev. 73, 346 - 368 (2017).

14. Burange, A. S., Gawande, M. B., Lam, F. L. Y., Jayaram, R. V. \& Luque, R. Heterogeneously catalyzed strategies for the deconstruction of high density polyethylene: plastic waste valorisation to fuels. Green Chem. 17, 146-156 (2015).

15. Chandrasekaran, S. R., Kunwar, B., Moser, B. R., Rajagopalan, N. \& Sharma, B. K. Catalytic thermal cracking of postconsumer waste plastics to fuels. 1. kinetics and optimization. Energy Fuels 29, 6068-6077 (2015).

16. Kaminsky, W. Polyolefins: 50 years after Ziegler and Natta I; Springer-Verlag: Berlin, Heidelberg (2013).

17. Souillart, L. \& Cramer, N. Catalytic $\mathrm{C}-\mathrm{C}$ bond activations via oxidative addition to transition metals. Chem. Rev. 115, 9410-9464 (2015).

18. Xia, Y. \& Dong, G. Temporary or removable directing groups enable activation of unstrained C-C bonds. Nat. Rev. Chem. 4, 600-614 (2020).

19. Sivaguru, P., Wang, Z., Zanoni, G. \& Bi, X. Cleavage of carbon-carbon bonds by radical reactions. Chem. Soc. Rev. 48, 2615-2656 (2019).

20. Morcillo, S. P. Radical-promoted $\mathrm{C}-\mathrm{C}$ bond cleavage: a deconstructive approach for selective functionalization. Angew. Chem., Int. Ed. 58, 14044-14054 (2019).

21. Shi, S.-H., Liang, Y. \& Jiao, N. Electrochemical Oxidation Induced Selective C-C Bond Cleavage. Chem. Rev. 121, 485-505 (2021).

22. Wu, X. \& Zhu, C. Recent advances in radical-mediated $\mathrm{C}-\mathrm{C}$ bond fragmentation 
of non-strained molecules. Chin. J. Chem. 37, 171-182 (2019).

23. Langeslay, R. R., Kaphan, D. M., Marshall, C. L., Stair, P. C., Sattelberger, A. P. \& Delferro, M. Catalytic applications of vanadium: a mechanistic perspective. Chem. Rev. 119, 2128-2191 (2019).

24. Yu, X.-Y., Chen, J.-R. \& Xiao, W.-J. Visible light-driven radical-mediated C-C bond cleavage/functionalization in organic synthesis. Chem. Rev. 121, 506-561 (2021). 25. Takaki, K., Yamamoto, J., Matsushita, Y., Morii, H., Shishido, T. \& Takehira, K. Oxidation of alkanes with dioxygen induced by visible light and $\mathrm{Cu}(\mathrm{II})$ and $\mathrm{Fe}(\mathrm{III})$ chlorides. Bull. Chem. Soc. Jpn. 76, 393-398 (2003).

26. Takaki, K., Yamamoto, J., Komeyama, K., Kawabata, T. \& Takehira K. Photocatalytic oxidation of alkanes with dioxygen by visible light and copper(II) and iron(III) chlorides: preference oxidation of alkanes over alcohols and ketones. Bull. Chem. Soc. Jpn. 77, 2251-2255 (2004).

27. Rosenthal, J., Luckett, T. D., Hodgkiss, J. M. \& Nocera, D. G. Photocatalytic oxidation of hydrocarbons by a bis-iron(III) $-\mu-$ oxo Pacman porphyrin using $\mathrm{O}_{2}$ and visible light. J. Am. Chem. Soc. 128, 6546-6547 (2006).

28. Li, S., Zhu, B., Lee, R., Qiao, B. \& Jiang Z. Visible light-induced selective aerobic oxidative transposition of vinyl halides using a tetrahalogenoferrate(III) complex catalyst. Org. Chem. Front. 5, 380-385 (2018).

29. Geng, S., Xiong, B., Zhang, Y., Zhang, J., He, Y. \& Feng, Z. Thiyl radical promoted iron-catalyzed-selective oxidation of benzylic $\mathrm{sp}^{3} \mathrm{C}-\mathrm{H}$ bonds with molecular oxygen. Chem. Commun. 55, 12699-12702 (2019).

30. Yu, H., Zhao, Q., Wei, Z., Wu, Z., Li, Q., Han, S. \& Wei, Y. Iron-catalyzed oxidative functionalization of $\mathrm{C}\left(\mathrm{sp}^{3}\right)-\mathrm{H}$ bonds under bromide-synergized mild conditions. Chem. Commun. 55, 7840-7843 (2019).

31. Urgoitia, G., SanMartin, R., Herrero, M. T. \& Domínguez E. Recent advances in homogeneous metal-catalyzed aerobic $\mathrm{C}-\mathrm{H}$ oxidation of benzylic compounds. Catalysts 8, 640 (2018).

32. Laudadio, G., Govaerts, S., Wang, Y., Ravelli, D., Koolman, H., Fagnoni, M., Djuric, S. \& Noël, T. Selective $\mathrm{C}\left(\mathrm{sp}^{3}\right)-\mathrm{H}$ aerobic oxidation enabled by decatungstate 
photocatalysis in flow. Angew. Chem., Int. Ed. 57, 4078-4082 (2018).

33. Xie, P., Xue, C., Shi, S. \& Du, D. Visible-light-driven selective air-oxygenation of $\mathrm{C}-\mathrm{H}$ bond via $\mathrm{CeCl}_{3}$ catalysis in water. ChemSusChem 14, 1-4 (2021).

34. Zhang, K., Chang, L., An, Q., Wang, X. \& Zuo, Z. Dehydroxymethylation of alcohols enabled by cerium photocatalysis. J. Am. Chem. Soc. 141, 10556-10564 (2019).

35. Chen, Y., Du, J. \& Zuo, Z. Selective $\mathrm{C}-\mathrm{C}$ bond scission of ketones via visible-light-mediated cerium catalysis. Chem 6, 266-279 (2020).

36. Wang, M., Lu, J., Li, L., Li, H., Liu, H. \& Wang, F. Oxidative C(OH)-C bond cleavage of secondary alcohols to acids over a copper catalyst with molecular oxygen as the oxidant. J. Catal. 348, 160-167 (2017).

37. Xu, L., Chen, Y., Shen, Z., Wang, Y. \& Li, M. $\mathrm{I}_{2} / \mathrm{Fe}\left(\mathrm{NO}_{3}\right)_{3} \cdot 9 \mathrm{H}_{2} \mathrm{O}-$ catalyzed oxidative synthesis of aryl carboxylic acids from aryl alkyl ketones and secondary benzylic alcohols. Tetrahedron Lett. 59, 4349-4354 (2018).

38. Liu, M., Zhang, Z., Song, J., Liu, S., Liu, H. \& Han, B. Nitrogen dioxide catalyzed aerobic oxidative cleavage of $\mathrm{C}(\mathrm{OH})-\mathrm{C}$ bonds of secondary alcohols to produce acids. Angew. Chem., Int. Ed. 58, 17393-17398 (2019).

39. Zhu, Y., Zhang, Z., Jin, R., Liu, J., Liu, G., Han, B. \& Jiao, N. DMSO-enabled selective radical $\mathrm{O}-\mathrm{H}$ activation of 1,3(4)-diols. Angew. Chem., Int. Ed. 59, 19851-19856 (2020).

40. Zhou, X., Ding, H., Chen, P., Liu, L., Sun, Q., Wang, X., Wang, P., Lv, Z. \& Li, M. Radical dehydroxymethylative fluorination of carbohydrates and divergent transformations of the resulting reverse glycosyl fluorides. Angew. Chem., Int. Ed. 59, 4138-4144 (2020).

41. Zhao, H., Fan, X., Yu, J. \& Zhu, C. Silver-catalyzed ring-opening strategy for the synthesis of $\beta-$ and $\gamma$-fluorinated ketones. J. Am. Chem. Soc. 137, 3490-3493 (2015). 42. Ren, R., Zhao, H., Huan, L. \& Zhu, C. Manganese-catalyzed oxidative azidation of cyclobutanols: regiospecific synthesis of alkyl azides by $\mathrm{C}-\mathrm{C}$ bond cleavage. Angew. Chem., Int. Ed. 54, 12692-12696 (2015).

43. Zhang, Z., Zhang, G., Xiong, N., Xue, T., Zhang, J., Bai, L., Guo, Q. \& Zeng, R. 
Oxidative $\alpha-\mathrm{C}-\mathrm{C}$ Bond cleavage of $2^{\circ}$ and $3^{\circ}$ alcohols to aromatic acids with $\mathrm{O}_{2}$ at room temperature via iron photocatalysis. Org. Lett. 23, 2915-2920 (2021).

44. Narayanam, J. M. R. \& Stephenson, C. R. J. Visible light photoredox catalysis: applications in organic synthesis. Chem. Soc. Rev. 40, 102-113 (2011).

45. Prier, C. K., Rankic, D. A. \& MacMillan, D. W. C. Visible light photoredox catalysis with transition metal complexes: applications in organic synthesis. Chem. Rev. 113, $5322-5363(2013)$.

46. Schultz, D. M. \& Yoon, T. P. Solar synthesis: prospects in visible light photocatalysis. Science 343, 1239176 (2014).

47. Jia, K. \& Chen, Y. Visible-light-induced alkoxyl radical generation for inert chemical bond cleavage/functionalization. Chem. Commun. 54, 6105-6112 (2018).

48. Jiang, H. \& Studer, A. Chemistry with $\mathrm{N}-$ centered radicals generated by single-electron transfer-oxidation using photoredox catalysis. CCS Chem. 1, 38-49 (2019).

49. Tsui, E., Wang, H. \& Knowles, R. R. Catalytic generation of alkoxy radicals from unfunctionalized alcohols. Chem. Sci. 11, 11124-11141 (2020).

50. Benzoic Acid Market Analysis, Market Size, Application Analysis, Regional Outlook, Competitive Strategies and Forecasts, 2016 To 2024; hexaresearch.com/researchreport/benzoic-acid-market

51. Lopez, L., Troisi, L., Rashid, S.M.K. \& Schaap A. P. Synthesis of 1,2-dioxetanes via 9,10-dicyanoanthracene-sensitized chain electron-transfer photooxygenations. Tetrahedron Lett. 30, 485-488 (1989).

52. Yi, H., Bian, C., Hu, X., Niu, L. \& Lei, A., Visible light mediated efficient oxidative benzylic $\mathrm{sp}^{3} \mathrm{C}-\mathrm{H}$ to ketone derivatives obtained under mild conditions using $\mathrm{O}_{2}$. Chem. Commun. 51, 14046-14049 (2015).

53. Lykakis, I. N. \& Orfanopoulos, M. Decatungstate-catalyzed photooxygenation of s2-phenylbutane and cumene via a free carbon-radical intermediate. Curr. Org. Chem. 13, 1737-1745 (2009).

54. Silverman, S. K. \& Foote, C. S. Singlet oxygen and electron-transfer mechanisms in the dicyanoanthracene-sensitized photooxidation of 2,3-diphenyl-1,4-dioxene. J. Am. 
Chem. Soc. 113, 7672-7675 (1991).

55. Schaap, A. P., Zaklika, K. A., Kaskar, B. \& Fung, L. W.-M. Mechanisms of photooxygenation. 2. Formation of 1.2-dioxetanes via 9,10-dicyanoanthracenesensitized electron-transfer processes. J. Am. Chem. Soc. 102, 389-391 (1980).

Acknowledgements P.H. would like to acknowledge financial support from the Department of Science and Technology of Guangdong Province (no. 2019QN01L151), the National Natural Science Foundation of China (no. 21821003), and Sun Yat-sen University. We thank Prof. Rong Zeng for helpful discussion.

Author Contributions M.W. made the initial discovery, carried out experiments and wrote the manuscript. J.W. carried out catalytic experiments and wrote the manuscript. Y.H. discussed the results and wrote the manuscript. P.H. designed and directed the project and wrote the manuscript.

\section{Competing interests}

There are no competing interests to declare

\section{Additional information}

Supplementary information is available for this paper.

Correspondence and requests for materials should be addressed to P.H. (hupeng8@mail.sysu.edu.cn) 